\title{
EDITORIAL
}

\section{EXERCISE, AGING AND FRAILTY: GUIDELINES FOR INCREASING FUNCTION}

\author{
R.A. MERCHANT ${ }^{1,2}$, J.E. MORLEY ${ }^{3}$, M. IZQUIERDO ${ }^{4,5}$
}

\begin{abstract}
1. Division of Geriatric Medicine, Department of Medicine, National University Hospital, Singapore, Singapore; 2. Department of Medicine, Yong Loo Lin School of Medicine, National University Singapore, Singapore, Singapore; 3. Division of Geriatric Medicine, Saint Louis University School of Medicine, St Louis, MO, USA; 4. Navarrabiomed, Complejo Hospitalario de Navarra (CHN)-Universidad Pública de Navarra (UPNA), Navarra Institute for Health Research (IdiSNA), Pamplona, Navarra, Spain; 5. CIBER of Frailty and Healthy Aging (CIBERFES), Instituto de Salud Carlos III, Madrid, Spain. Corresponding author: Mikel Izquierdo, PhD, Department of Health Sciences, Public University of Navarra, Av. De Barañain s/n 31008 Pamplona (Navarra) Spain, Tel + 34948 417876, mikel.izquierdo@ gmail.com
\end{abstract}

Key words: Exercise, frailty, elderly, function.

The population is ageing worldwide at a phenomenal pace from 900 million $\geq 60$ years old in 2015 to 2 billion in 2050 (1). The longer lifespan is due to advancement in public health, medical, social and economic development. However, healthspan has been slow to improve in most countries where the last decade of life is spent in poor health (2). Aging is associated with declines in functional capacity, and preserving function including lengthening healthspan is an increasingly important challenge for countries with a fast aging population. The World Report on Ageing and Health by World Health Organisation (WHO) defines healthy ageing as the process of developing and maintaining functional ability that enables wellbeing (3). The interaction between individual's intrinsic capacity and environmental characteristics are crucial to achieve the optimum trajectory which can be modified to maintain a person's functional ability and intrinsic capacity throughout the life course. We assert that greater knowledge of the effects of the exercise interventions on age-related amelioration of intrinsic capacity domains (i.e locomotion, vitality, cognition, psychological, sensory) that is present in the frail will allow a more coherent and holistic approach to treatment of the frail. This Viewpoint emphasizes the idea that the physiological bases underlying the assertions that exercise treatment of frailty directed at increasing muscle mass by pharmaceuticals in order to treat symptoms of frailty is an example of current medical, scientific and pharmaceutical industrial lack of appreciation of the role of exercise as a therapeutic agent having a major role both in the treatment and prevention not only of disease but also in functional capacity (4, 5).

Physical inactivity and social isolation accompanying ageing leads to decline in muscle strength, muscle mass and accelerates frailty, worsens chronic health issues, including hypertension, cardiovascular and cerebrovascular disease, diabetes, depression and dementia. Presently, there are no pharmacological agents (or combinations) or care standards known to slow down ageing. Physical activity including exercise training have been shown to influence key drivers of ageing even in the oldest-old including chronic inflammation, mitochondrial dysfunction, myokine release, autophagy, oxidative damage and insulin-like growth factor signaling $(4,6,7)$. Exercise improves physical function and quality of life, reduces the burden of non-communicable diseases and premature overall mortality including cause specific mortality from cardiovascular disease, cancer, and chronic lower respiratory tract diseases (8-10). WHO has recently released guidelines on Physical Activity and Sedentary Behaviour strongly recommending moderate or greater intensity multicomponent physical activity on $\geq 3$ days a week with emphasis on functional balance and strength training for improving functional capacity and preventing falls in older adults and those with chronic diseases (11). General physical exercise guidelines often focus on prevention of pathologies. Population level preventive efforts ideally before the onset of functional decline have been tried out and tested in many countries (12-16). Unfortunately, a limited amount of physical exercise strategies are planned to minimize exercise-related impact on function and/or ability to perform ADLs or in other domains of intrinsic capacity on older population, probably related to the scarcity of research in the area (4).

Aging is a risk factor for chronic diseases, frailty and dementia. Similarly, chronic disease can accelerate biological aging, frailty and dementia (17). Frailty is a dynamic clinical state of decreased functional reserve which increases individual's vulnerability to develop negative health-related events such as disability and/or mortality when exposed to external stressor (18). It is associated with a variety of negative outcomes including cognitive decline, falls, fear of falling, hospitalisation, increased healthcare utilisation, polypharmacy, institutionalisation, and mortality $(19,20)$. Frailty is not synonymous with disability or comorbidities, and almost half of frail older adults are still independent. Screening for frailty across a variety of healthcare settings including intermediate and long-term care settings and community is crucial to enable targeted personalised management $(16,18,21-23)$. A few of the commonly used frailty screening tools include the Fried's Frailty Phenotype often known as the Cardiovascular Health Study (CHS) index, FRAIL scale, Frailty Index of Accumulative Deficits (FI-CD) and Clinical Frailty Scale (2427). All of these tools are distinct but capture over-lapping 


\section{Figure 1}

The Trajectory of Healthy Ageing, Frailty and Acute Illness / Hospital associated Disability

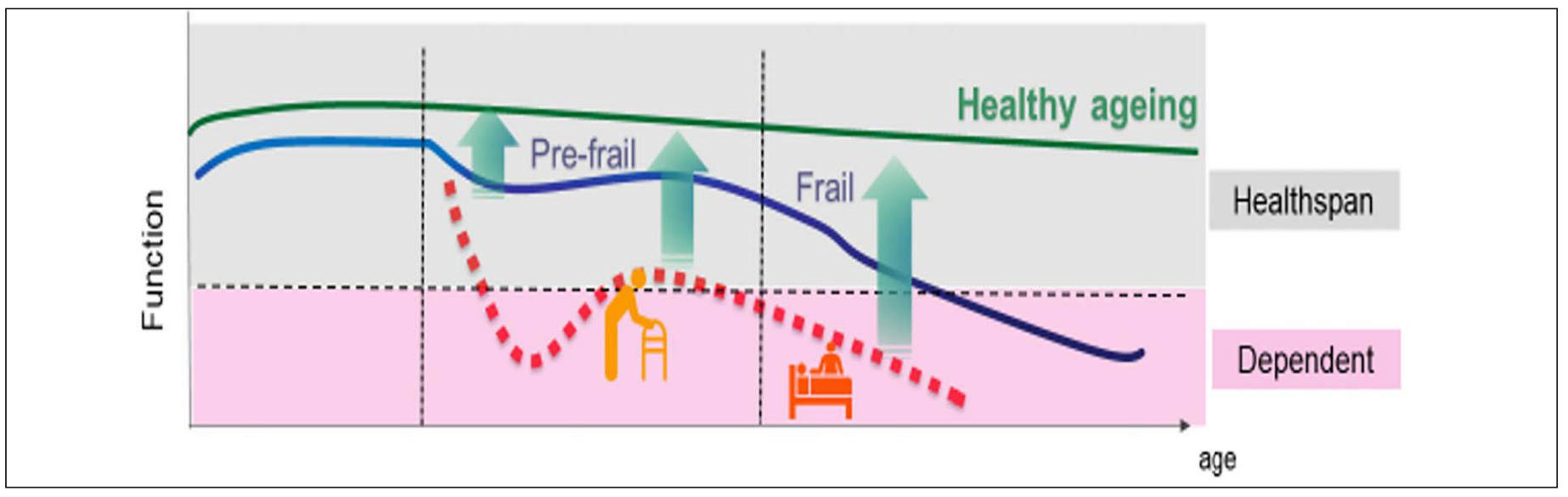

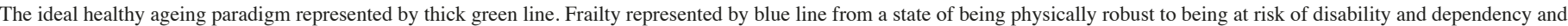
dying. Acute illness and / or hospitalization represented by dotted red line. Green arrows highlights benefit of exercise training and multicomponent interventions.

constructs and predicts poor functional outcomes. The FRAIL scale is fast, simple and convenient to use at the population level and has been validated in many countries (28). In a recent systematic review, the pooled prevalence of prefrailty ranged between $35 \%$ to $50 \%$, and frailty $7 \%$ to $12 \%$, increasing to $26 \%$ in those aged over 85 years $(29,30)$. Sarcopenia, the loss of muscle mass and strength, is the major cause of frailty (31, 32). One of the main challenges for the future is to use these scales which usually focus on identification of pathology or pathophysiology that accompany frailty to also monitor intrinsic domains (i.e locomotion, vitality, cognition, psychological, sensory) after multidomain non-pharmacological interventions (for example exercise or cognitive interventions).

Given the multidimensional nature of frailty comprising of physical, cognitive and social domains, reversing, preventing or slowing down progression of frailty requires a targeted multicomponent approach including physical exercise, management of polypharmacy, falls, nutrition, loneliness, cognitive impairment and depression for reversal and/or prevention of frailty $(23,33-35)$. Healthy and, even more so, chronically ill older adults are candidates for physical exercise strategies that will lessen the burden of comorbidity, disability and premature death caused by incident disease. A 10-year community-based multicomponent interventions (physical, nutrition and social encompassing group-based activities) in Kusatsu (Japan) improved functional status, reduced annual disability incidence, and prolonged active life expectancy at age 70 (36). Exercise interventions in nursing homes and longterm care facilities improve muscle strength and function (34, 35, 37-39). These benefits are lost after training cessation (35), especially in frail elderly patients with dementia after long-term physical restraint. In a recent study (38) on older adults $(<75$ years) living in nursing homes the Vivifrail multicomponent tailored exercise program (http://vivifrail.com/resources/) was very effective in the short-term (4 weeks) and prevented severe functional decline and strength loss in institutionalized older (i.e. physical frailty reversion and recovery of autonomy). Multicomponent exercise face-to-face interventions would seem advisable as an essential activity to protect older adults from severe functional decline (38).

The community-based approach is the best way forward and physical exercise is one of the main interventions with systemic effect proven to improve physical impairment related to frailty (low body mass, strength, mobility, physical activity level, energy) (5). Inter-individual variability and dose-response heterogeneity (subject-by-training interaction; 'individual response') in response to exercise is critical for defining threshold and optimal levels of activity that are necessary for health promotion and disease management. The type, intensity, frequency and duration of physical continues to be an area of research in frail older person (40). Twenty to thirty years ago, studies showed clear evidence that prolonged moderateto-high intensity strength-training (i.e. $60-80 \%$ of maximum strength, 2-3 sets of 8-12 repetitions) would lead to large gains in maximal strength and power, muscle mass and functional ability $(41,42)$. Also, power training, including exercises in which daily activities are stimulated, are recommended including to optimize the functional capacity of frail individuals (43). Unfortunately, dose-response relationships between changes in fitness, function and better health outcomes have been defined for some, but certainly not for all, diseases and syndromes $(44,45)$. Some modalities or doses of exercise that are promoted for older adults (mild calisthenics, slow-paced walking) have little or no discernible effects on physical fitness but may possibly yield benefits in some domains. This area of investigation is critical for defining threshold and optimal levels of activity that are necessary for health promotion and disease management. Exercise and medical researchers have recognized the substantial variability in patient response to physical exercise interventions and have sought to understand these differences. Recently, it was reported that older patients performing an individualized exercise intervention showed 
Figure 2

VIVIFRAIL Multicomponent Physical Exercise Program to Prevent Frailty and the Risk of Falls. Example of exercise wheels for each functional level that include the exercises, series and repetitions that should be done every week. Available at (53)

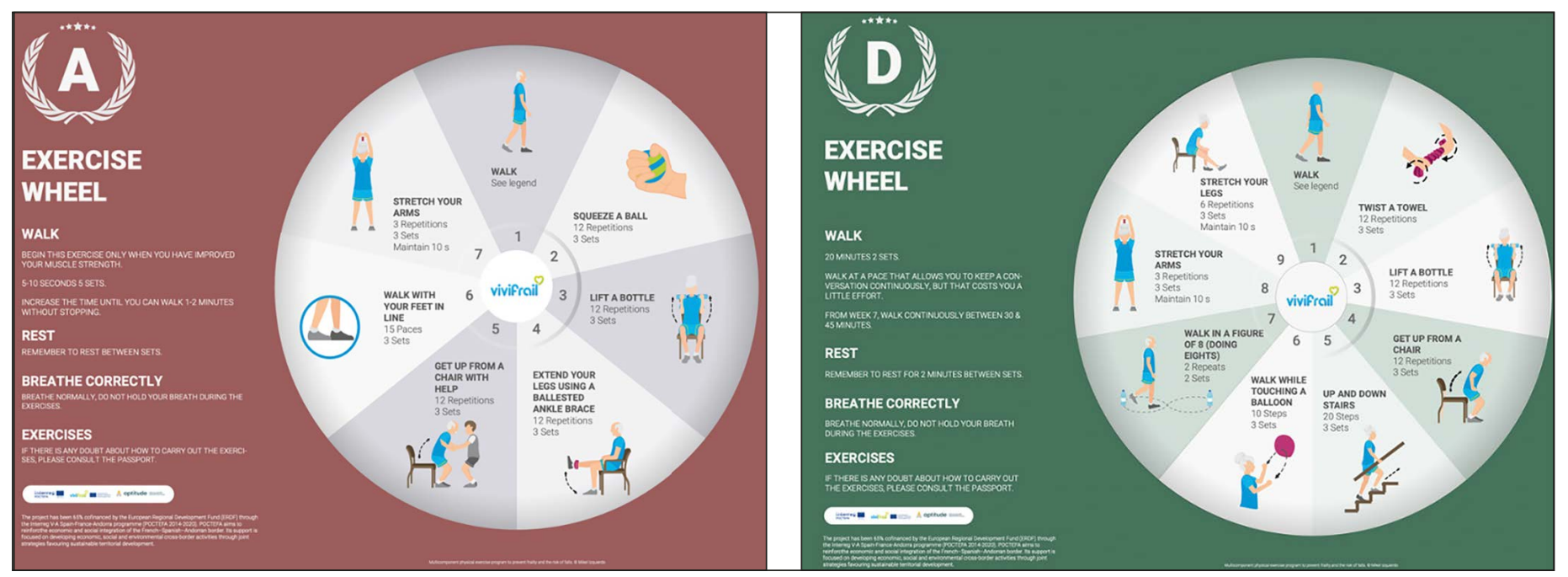

An example of exercise wheel type A (frail) for an older people who can walk with difficulty or help and type D for a robust person. (free download from http://vivifrail.com/resources/). Additionally, an App to perform the Vivifrail Test and to follow the exercise program corresponding to the degree of frailty and risk of falls is also available on Google Play or the App Store.

\section{Figure 3}

Poster to promote the participation in the VIVIFRAIL program of elderly people who come to centers and consultations. Step by step the way to participate in the exercise program that best suits the initial functional status is described. Available at (53)

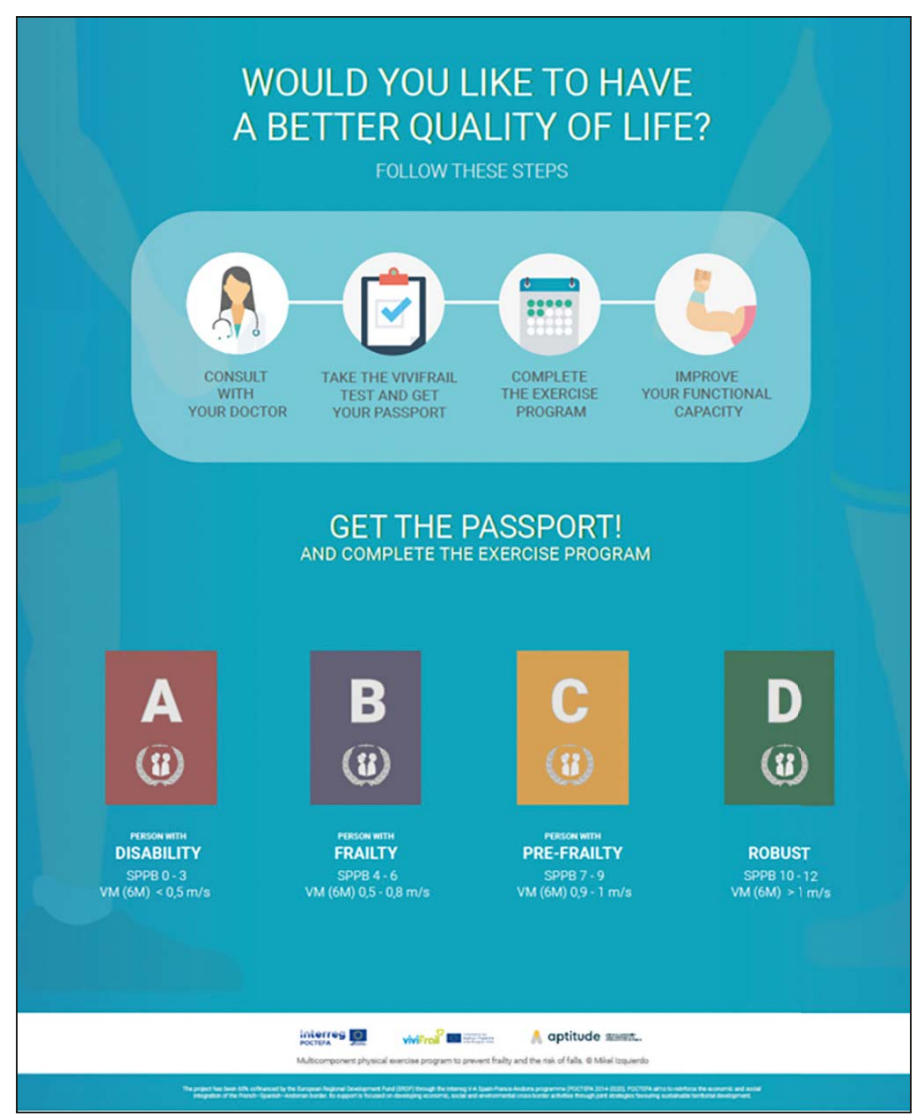

a higher prevalence of responders and a lower prevalence of non-responders and adverse-responders for functional capacity, muscle strength, and cognitive function than those who were treated with usual care during acute hospitalization (46). An adverse response on functional capacity in older medical patients to physical exercise or usual care during hospitalization was associated with mortality at one year post-discharge. Moreover, the functional status presented at admission seems to be a cornerstone in the trajectory of patients during hospital stay and even more so at follow-up. These findings support the need for a shift from the traditional disease-focused approach in hospital acute care to one that recognizes functional status including gait speed as a clinical vital sign $(46,47)$.

Pre-frail and frail older adults are particularly vulnerable to adverse effects of hospitalisation especially functional decline and delirium as shown in Figure 1. In hospital, supervised exercise interventions have been shown to be safe and effective in attenuating functional decline and preventing cognitive decline (48-51). A multicomponent exercise program performed twice daily supervised and unsupervised for 20 minutes each during 5 to 7 consecutive days has shown to improve physical function with indirect effect on cognitive function (51). The exercises found to be beneficial included progressive resistance, balance, and walking training exercises aiming at 2 to 3 sets of 8 to 10 repetitions tailored to the individual's functional capacity with a load equivalent to $30 \%$ to $60 \%$ of the 1-repetition maximum. The type of exercises were based on clinical physical exercise guide, Vivifrail (http://vivifrail.com/ resources/), involving mainly lower-limb muscles (squats rising from a chair, leg press, and bilateral knee extension), upper body (seated bench press), balance and gait re-training (semi tandem line walking, foot standing, stepping practice, walking 


\section{EXERCISE, AGING AND FRAILTY: GUIDELINES FOR INCREASING FUNCTION}

with small obstacles, proprioceptive exercises on unstable surfaces such as foam pads sequence, weight transfer from 1 leg to the other) $(42,52)$. Vivifrail has individual prescription passports for older adults depending on the older person's functional capacity level (serious limitation, moderate limitation and slight limitation as evaluated by the SPPB and a walking speed test) and the risk of falling (www.vivifrail.com) which can be implemented during unsupervised sessions (Figure 2 and Figure 3) (53).

Healthy ageing and lengthening healthspan should be a public health priority for every country. Physical activity including exercise training of low and/or moderate intensity have been found to be effective and safe in improving physical function even in the oldest old and frail older adults $(54,55)$. Community embedded program incorporating dual-task exercse to promote healthy ageing like the Healthy Ageing Promotion Program for You (HAPPY) has shown to improve cognition, physical function and frailty status, reduce social isolation and improve perceived health. Multicomponent physical exercise programs tailored to the persons functional capacity are fundamental to maintaining mobility, musculoskeletal function and optimal function of other body systems: neurological, cardiovascular, respiratory, and endocrine. However, a new era in the precision of the exercise prescription should also consider a priori the optimal exercise intervention being the more timeefficient but eliciting a smaller proportion of non-responder participants. Yet, exercise has not become fully integrated into usual primary or geriatric medicine practice and is still virtually absent from the core training of most medical doctors and other healthcare professionals.

Disclosures: The authors declare no conflicts of interest.

\section{References}

1. World Health Organization [WHO] (2018). Ageing and Health

2. Crimmins EM. Lifespan and Healthspan: Past, Present, and Promise. The Gerontologist. 2015;55(6):901-911

3. Beard JR, Officer A, de Carvalho IA, et al. The World report on ageing and health: a policy framework for healthy ageing. Lancet. 2016;387(10033):2145-2154

4. Izquierdo M, Morley JE, Lucia A. Exercise in people over 85. BMJ. 2020;368:m402.

5. Lazarus NR, Izquierdo M, Higginson IJ, Harridge SDR. Exercise deficiency diseases of aging: The primacy of exercise and muscle strengthening as first-line therapeutic agents to combat frailty. J Am Med Dir Assoc 2018;19:741-743.

6. Angulo J, El Assar M, Álvarez-Bustos A, Rodríguez-Mañas L. Physical activity and exercise: Strategies to manage frailty. Redox Biology. 2020;35:101513.

7. Valenzuela PL, Castillo-Garcia A, Morales JS, et al. Physical Exercise in the Oldest Old. Compr Physiol. 2019;9(4):1281-1304.

8. Kujala UM. Is physical activity a cause of longevity? It is not as straightforward as some would believe. A critical analysis. British Journal of Sports Medicine. 2018;52(14):914-918

9. Stensvold D, Viken H, Steinshamn SL, et al. Effect of exercise training for five years on all cause mortality in older adults - the Generation 100 study: randomised controlled trial. BMJ. 2020;371:m3485

10. Zhao M, Veeranki SP, Magnussen CG, Xi B. Recommended physical activity and all cause and cause specific mortality in US adults: prospective cohort study. BMJ. 2020;370:m2031.

11. World Health Organisation: WHO Guidelines on Physical Activity and Sedentary Behaviour. 2020

12. Osuka Y, Kojima N, Yoshida Y, et al. Exercise and/or Dietary Varieties and Incidence of Frailty in Community-Dwelling Older Women: A 2-Year Cohort Study. J Nutr Health Aging. 2019;23(5):425-430.

13. Sadjapong U, Yodkeeree S, Sungkarat S, Siviroj P. Multicomponent Exercise
Program Reduces Frailty and Inflammatory Biomarkers and Improves Physical Performance in Community-Dwelling Older Adults: A Randomized Controlled Trial. Int J Environ Res Public Health. 2020;17(11).

14. Izquierdo M, Rodriguez-Mañas L, Sinclair AJ, Vivifrail Investigators Group. What is new in exercise regimes for frail older people - How does the Erasmus Vivifrail Project take us forward? J Nutr Heal Aging. Springer-Verlag France; 2016;20:736-7.

15. Casas-Herrero A, Anton-Rodrigo I, Zambom-Ferraresi F, et al. Effect of a multicomponent exercise programme (VIVIFRAIL) on functional capacity in frail community elders with cognitive decline: study protocol for a randomized multicentre control trial. Trials. 2019;20(1):362.

16. Lundy J, Hayden D, Pyland S, Berg-Weger M, Malmstrom TK, Morley JE. An AgeFriendly Health System. J Am Geriatr Soc. 2020.

17. Hanlon P, Nicholl BI, Jani BD, Lee D, McQueenie R, Mair FS. Frailty and pre-frailty in middle-aged and older adults and its association with multimorbidity and mortality: a prospective analysis of 493737 UK Biobank participants. Lancet Public Health. 2018;3(7):e323-e332.

18. Morley JE, Vellas B, van Kan GA, et al. Frailty consensus: a call to action. J Am Med Dir Assoc. 2013;14(6):392-397.

19. Tan LF, Lim ZY, Choe R, Seetharaman S, Merchant R. Screening for Frailty and Sarcopenia Among Older Persons in Medical Outpatient Clinics and its Associations With Healthcare Burden. J Am Med Dir Assoc. 2017;18(7):583-587.

20. Merchant RA, Chen MZ, Wong BLL, et al. Relationship Between Fear of Falling, Fear-Related Activity Restriction, Frailty, and Sarcopenia. J Am Geriatr Soc. 2020.

21. Merchant RA, Hui RJY, Kwek SC, et al. Rapid Geriatric Assessment Using Mobile App in Primary Care: Prevalence of Geriatric Syndromes and Review of Its Feasibility. Frontiers in Medicine. 2020;7(261).

22. Merchant RA, Chen MZ, Tan LWL, Lim MY, Ho HK, van Dam RM. Singapore Healthy Older People Everyday (HOPE) Study: Prevalence of Frailty and Associated Factors in Older Adults. J Am Med Dir Assoc. 2017.

23. Ruiz JG, Dent E, Morley JE, et al. Screening for and Managing the Person with Frailty in Primary Care: ICFSR Consensus Guidelines. The journal of nutrition, health \& aging. 2020;24(9):920-927.

24. Mitnitski AB, Mogilner AJ, Rockwood K. Accumulation of Deficits as a Proxy Measure of Aging. TheScientificWorldJOURNAL. 2001;1:321027.

25. Rockwood K, Song X, MacKnight C, et al. A global clinical measure of fitness and frailty in elderly people. Canadian Medical Association Journal. 2005;173(5):489495.

26. Fried LP, Tangen CM, Walston J, et al. Frailty in older adults: evidence for a phenotype. J Gerontol A Biol Sci Med Sci. 2001;56(3):M146-156.

27. Malmstrom TK, Miller DK, Morley JE. A comparison of four frailty models. J Am Geriatr Soc. 2014;62(4):721-726.

28. Woo J, Yu R, Wong M, Yeung F, Wong M, Lum C. Frailty Screening in the Community Using the FRAIL Scale. J Am Med Dir Assoc. 2015;16(5):412-419.

29. Collard RM, Boter H, Schoevers RA, Oude Voshaar RC. Prevalence of frailty in community-dwelling older persons: a systematic review. J Am Geriatr Soc 2012;60(8):1487-1492

30. O'Caoimh R, Sezgin D, O'Donovan MR, et al. Prevalence of frailty in 62 countries across the world: a systematic review and meta-analysis of population-level studies. Age Ageing. 2020.

31. Sanford AM, Morley JE, Berg-Weger M, et al. High prevalence of geriatric syndromes in older adults. PLoS One. 2020 Jun 5;15(6):e0233857.

32. Bauer J, Morley JE, Schols AMWJ, et al. Sarcopenia: A Time for Action. An SCWD Position Paper. J Cachexia Sarcopenia Muscle. 2019 Oct;10(5):956-961

33. Tarazona-Santabalbina FJ, Gómez-Cabrera MC, Pérez-Ros P, et al. A Multicomponent Exercise Intervention that Reverses Frailty and Improves Cognition, Emotion, and Social Networking in the Community-Dwelling Frail Elderly: A Randomized Clinical Trial. Journal of the American Medical Directors Association. 2016;17(5):426-433.

34. Cadore EL, Casas-Herrero A, Zambom-Ferraresi F, Idoate F, Millor N, Gómez M, Rodriguez-Mañas L, Izquierdo M. Multicomponent exercises including muscle power training enhance muscle mass, power output, and functional outcomes in institutionalized frail nonagenarians. Age (Dordr). 2014 Apr;36(2):773-85. doi: 10.1007/s11357-013-9586-z. Epub 2013 Sep 13.

35. Cadore EL, Moneo AB, Mensat MM, Muñoz AR, Casas-Herrero A, RodriguezMañas L, Izquierdo M. Positive effects of resistance training in frail elderly patients with dementia after long-term physical restraint. Age (Dordr). 2014 Apr;36(2):80111. doi: 10.1007/s11357-013-9599-7. Epub 2013 Nov 16

36. Shinkai S, Yoshida H, Taniguchi Y, et al. Public health approach to preventing frailty in the community and its effect on healthy aging in Japan. Geriatr Gerontol Int. 2016;16 Suppl 1:87-97.

37. Yoshiko A, Kaji T, Sugiyama H, et al. Twenty-Four Months' Resistance and Endurance Training Improves Muscle Size and Physical Functions but Not Muscle Quality in Older Adults Requiring Long-Term Care. J Nutr Health Aging. 2019;23(6):564-570

38. Courel-Ibanez J, Pallares JG, et al. Supervised Exercise (Vivifrail) Protect Institutionalized Older Adults Against Severe Functional Decline After 14 Weeks of COVID Confinement. J Am Med Dir Assoc. 2021 Jan;22(1):217-219.e2. doi: 10.1016/j.jamda.2020.11.007. Epub 2020 Dec 6. 


\section{THE JOURNAL OF NUTRITION, HEALTH \& AGING}

39. Brown M, Sinacore DR, Ehsani AA, Binder EF, Holloszy JO, Kohrt WM. Lowintensity exercise as a modifier of physical frailty in older adults. Arch Phys Med Rehabil. 2000;81(7):960-965.

40. Silva RB, Aldoradin-Cabeza H, Eslick GD, Phu S, Duque G. The Effect of Physical Exercise on Frail Older Persons: A Systematic Review. J Frailty Aging. 2017;6(2):91-96.

41. Izquierdo M, Ibanez J, K HA, Kraemer WJ, Larrion JL, Gorostiaga EM. Once weekly combined resistance and cardiovascular training in healthy older men. Med Sci Sports Exerc. 2004;36(3):435-443.

42. Fiatarone MA, Marks EC, Ryan ND, Meredith CN, Lipsitz LA, Evans WJ. Highintensity strength training in nonagenarians. Effects on skeletal muscle. Jama 1990;263(22):3029-3034.

43. Izquierdo M, Häkkinen K, Ibañez J, Garrues M, Antón A, Zúñiga A, Larrión JL, Gorostiaga EM. Effects of strength training on muscle power and serum hormones in middle-aged and older men. J Appl Physiol (1985). 2001 Apr;90(4):1497-507. doi: 10.1152/jappl.2001.90.4.1497

44. Álvarez C, Ramírez-Campillo R, Ramírez-Vélez R, Izquierdo M. Effects and prevalence of nonresponders after 12 weeks of high-intensity interval or resistance training in women with insulin resistance: A randomized trial. J Appl Physiol. 2017;122(4). doi:10.1152/japplphysiol.01037.2016

45. Ramírez-Vélez R, Lobelo F, Izquierdo M. Exercise for Disease Prevention and Management: A Precision Medicine Approach. J Am Med Dir Assoc. 2017;18(7) doi:10.1016/j.jamda.2017.04.012

46. Sáez de Asteasu ML, Martínez-Velilla N, Zambom-Ferraresi F, Casas-Herrero Á, Cadore EL, Ramirez-Velez R, et al. Inter-individual variability in response to exercise intervention or usual care in hospitalized older adults. J Cachexia Sarcopenia Muscle [Internet]. 2019 [cited 2019 Sep 5];jcsm.12481. Available from: https://onlinelibrary. wiley.com/doi/abs/10.1002/jcsm.12481

47. Merchant RA, Goh J, Chan YH, et al. Slow Gait, Subjective Cognitive Decline and Motoric Cognitive RISK Syndrome: Prevalence and Associated Factors in Community Dwelling Older Adults. J Nutr Health Aging 2021;25(1):48-56
48. Saez de Asteasu ML, Martinez-Velilla N, Zambom-Ferraresi F, Ramirez-Velez R, Garcia-Hermoso A, Izquierdo M. Cognitive Function Improvements Mediate Exercise Intervention Effects on Physical Performance in Acutely Hospitalized Older Adults. J Am Med Dir Assoc. 2020 Sep 30:S1525-8610(20)30731-3. doi: 10.1016/j. jamda.2020.08.024. Epub ahead of print. PMID: 33011095.

49. Valenzuela PL, Morales JS, Castillo-Garcia A, et al. Effects of exercise intervention on the functional status of acutely hospitalised older adults: A systematic review and meta-analysis. Ageing Res Rev. 2020;61:101076.

50. Wang R, Liang Y, Jiang J, Chen M, Li L, Yang H, Tan L, Yang M.J Nutr Health Aging. 2020;24(10):1087-1093 Effectiveness of a Short-Term Mixed Exercise Program for Treating Sarcopenia in Hospitalized Patients Aged 80 Years and Older: A Prospective Clinical Trial.

51. Martinez-Velilla N, Casas-Herrero A, Zambom-Ferraresi F, et al. Effect of Exercise Intervention on Functional Decline in Very Elderly Patients During Acute Hospitalization: A Randomized Clinical Trial. JAMA Intern Med. 2019;179(1):28 36.

52. Izquierdo M, Casas-Herrero A, Zambom-Ferraresi F, Martínez-Velilla N, AlonsoBouzón C, Rodriguez-Manas L. Multicomponent physical exercise program VIVIFRAIL [Internet]. Pamplona; 2019 [cited 2020 Dec 28th]. Available from: http://vivifrail.com/wp-content/uploads/2019/11/VIVIFRAIL-ENG-Interactivo.pdf

53. Izquierdo M. Multicomponent physical exercise program VIVIFRAIL [Internet]: Exercise wheels by functional level type. Available at: http://vivifrail.com/resources (Accessed 27th December 2020).

54. Beckwee D, Delaere A, Aelbrecht S, et al. Exercise Interventions for the Prevention and Treatment of Sarcopenia. A Systematic Umbrella Review. J Nutr Health Aging. 2019;23(6):494-502.

55. García-Hermoso A, Ramirez-Vélez R, Sáez de Asteasu ML, Martínez-Velilla N, Zambom-Ferraresi F, Valenzuela PL, Lucia A, Izquierdo M. Safety and Effectiveness of Long-Term Exercise Interventions in Older Adults: A Systematic Review and Meta-analysis of Randomized Controlled Trials. Sports Med. 2020 Jun;50(6):1095 1106 